\title{
Sémiotique et publicité en \\ classe de FLE : une nouvelle approche visant le niveau A2 du Cadre Européen Commun de Référence pour les Langues ${ }^{1}$
}

\section{(Semiótica y publicidad en la clase de FLE: nuevo enfoque para el nivel A2 del Marco Común de Referencia Europeo para las Lenguas)}

\author{
Marcela Ortiz Cabezas² \\ Universidad Nacional, Costa Rica
}

\section{RÉSUME}

Il propose un entraînement à l'analyse du discours et une réflexion sur son importance, à travers un dispositif adressé aux élèves ayant acquis le niveau A1 du CECRL. Ce dispositif veut constituer aussi une proposition au-delà de la communication évidente entre locuteurs pour devenir un entraînement à l'analyse du discours à partir d'une optique souple et pratique, adaptée aux jeunes apprenants.

\section{RESUMEN}

Se propone un entrenamiento para el análisis del discurso y una reflexión sobre su importancia, mediante un dispositivo dirigido a estudiantes con el nivel A1 del CECRL. Este dispositivo es también una propuesta que se basa en la comunicación evidente entre locutores, para convertirse en un

1 Recibido: 19 de agosto de 2013; aceptado: 30 de setiembre de 2013. Elaborado con base en la ponencia presentada por la autora en el IV Congreso Internacional de Lingüística Aplicada, llevado a cabo en mayo de 2013, en el Campus Omar Dengo, de la Universidad Nacional de Costa Rica.

2 Escuela de Literatura y Ciencias del Lenguaje. Correo electrónico: marcela_ortizc@yahoo.es 
entrenamiento para el análisis del discurso, desde una perspectiva flexible y práctica, adaptada para los jóvenes aprendientes.

Mots-clés: enseignement du FLE, sémiotique, analyse du discours, publicité, iconotexte

Palabras clave: enseñanza de FLE, semiótica, análisis del discurso, publicidad, iconotexto

\section{Introduction}

Cet article présente une nouvelle façon d'aborder le sujet de la publicité en classe de FLE, qui prétend élargir les possibilités d'analyse, pas seulement du point de vue communicatif, mais aussi du point de vue linguistique et sémantique. Le but c'est d'éveiller chez les apprenants l'esprit critique, à travers l'analyse du discours publicitaire, pour leur fournir un bon outil.

L'étude présente une analyse du discours de la publicité du point de vue sémiotique, qui vise à mettre en relief l'importance de reconnaître l'intention du discours derrière les composantes linguistiques pour des apprenants ayant acquis le niveau A1 du CECRL ${ }^{3}$. Le dispositif qui en résulte veut constituer aussi une proposition au-delà de la communication évidente entre locuteurs pour devenir un entrainement à l'analyse du discours dès une optique souple et pratique adaptée aux jeunes apprenants. Il ne prétend pas être exhaustif, ni avoir le dernier mot. Il n'est qu'une expérience à partager dans l'enseignement de la langue française.

La recherche commence par un bref survol des clés pour la « lecture » de la publicité et le motif déclencheur du travail. Nous abordons la notion de publicité suggestive et ses stratégies, comme un des concepts de base de la proposition. Ensuite, nous nous sommes servies principalement des apports de plusieurs théoriciens de différentes origines : Roland Barthes, Frédéric Lambert et Patrick Charaudeau,

3 CERCL est la dénomination courante pour le Cadre Européen Commun de Référence pour les Langues. 
des français ; Helena Ivranova-Glédel, une russe ; et Gilles Lugrin, un suisse ; afin d'établir la notion d'iconotexte qui devient le concept fondateur d'un dispositif de travail d'analyse du discours publicitaire et les notions sémiologiques fondatrices de la proposition. Le dispositif complet proposé vise une application intégrée dans les cours de langue dispensés pour la dixième année du lycée. Trois étapes sont prévues en utilisant un ensemble de sept fiches pédagogiques.

\section{Antécédents}

La publicité est présente dans presque toutes les activités et tous les moments de nos vies. Tout est sujet de publicité et elle est chargée de sens et de messages. Les jeunes sont exposés constamment de façon directe ou indirecte et qu'ils le veuillent ou non, ils sont tous influencés. La publicité est présente dans les rues, aux arrêts d'autobus, dans les vêtements et on la trouve aussi, bien sûr, dans les multimédia comme internet.

Grâce aux satellites, et aux chaînes de télévision, qui réunissent le monde en une seconde, la publicité est devenue une question d'offres et de besoins-créés, généralisés tout autour du monde. C'est un phénomène global dont on peut même dire que nous sommes tous concernés.

Jusqu'à présent, la publicité a été étudiée prioritairement du point de vue communicatif, selon le schéma de Jakobson. Voilà pourquoi nous proposons un type de lecture un peu plus approfondie, car le but et l'origine de la publicité a une charge économique impressionnante...

\section{La publicité et l'économie}

Essayer de calculer les chiffres d'investissement mondial en matière de publicité est pratiquement impossible. Il faut compter ce qui est réglé par les médias, sans oublier les dépenses en salaires des spécialistes, des créatifs, des exécutifs, ainsi que les budgets destinés à la production des matériaux et toutes les pièces du marché. 
Néanmoins, $d$ 'après la firme française Greenwich Consulting, (Le monde, 31/01/08), pendant l'année 2007, s'y sont investis, mondialement, 475.7 milliards de dollars distribués comme suit :

- Presse: $+3 \%$ (183,7 milliards)

- Télévision: $+7,1 \%$ (180,3 milliards)

- Internet: $+23,9 \%$ (44,6 milliards)

- Radio: $+3,6 \%$ (37,8 milliards)

- Affichage: $+8 \%$ (27,1 milliards)

- Cinéma: $+10 \%$ (2,2 milliards)

$\mathrm{Au}$ début du XXI $\mathrm{x}^{\mathrm{e}}$ siècle, chaque français, « est en moyenne soumis à environ huit cents messages publicitaires par jour : télé, affiches, radio, ainsi que contact avec autrui (port de vêtement de marque et discussions sur des sujets... dont la publicité apparaît »; < www.techno-sciences.net>.

Selon le Service d' infographie du Figaro (20/07/2012 à 20:23) en France, « le montant total des investissements avoisine 14 milliards d'euros bruts. Or, au Costa Rica, la situation n'est pas très différente. Les chiffres d'investissement publicitaire parlaient d'un peu moins de 100 millions de dollars par an, en 2007. ${ }^{4}$ Et en ce qui concerne les années 2010 et 2011, nous transcrivons la grille comparative par type de média, facilité par l'Association costaricienne d'agences publicitaires (ASCAP), ${ }^{5}$ réalisée par 1'entreprise d'études publicitaires Media Guru. Les chiffres se montrent en millers de dollars.

4 Consulté en : <http://www.forospanama.com/showthread.php?t=11754>.

5 ASCAP, 18/10/2012. 


\begin{tabular}{|l|r|r|r|r|}
\hline TYPE DE MÉDIA & $2010-\mathrm{S} 1$ & SH1 & $2011-\mathrm{S} 1$ & SH2 \\
\hline Television & 88.105 .0 & 44 & 100.632 .2 & 45 \\
\hline Journaux & 45.352 .8 & 25 & 55.922 .8 & 23 \\
\hline Radio & 33.124 .8 & 16 & 37.210 .7 & 17 \\
\hline Magazines & 14.679 .7 & 7 & 16.795 .6 & 8 \\
\hline Pub. exterieure & 11.812 .7 & 7 & 14.724 .8 & 6 \\
\hline Autres & 2.864 .6 & 1 & 3.129 .5 & 2 \\
\hline Total général & 195.940 & 100.1 & 228.416 & 99.9 \\
\hline
\end{tabular}

Source: Média Guru

De plus, certains événements contribuent à faire de la publicité une très bonne affaire. Par exemple, tous les quatre ans elle prend un nouvel élan avec les campagnes électorales et cela arrive du moins dans tout le continent américain : la publicité est devenue le principal moyen de diffusion des partis politiques.

On trouve aussi le phénomène sportif, tous les quatre ans, lui aussi, avec par exemple, la préparation de la Coupe du Monde de football, qui pousse la publicité au premier rang. En 2006 on pouvait lire dans nacion.com les déclarations du PDG d'études publicitaires de Media Guru, Francisco Correa, affirmant que « la publicité concernant le Mondial d'Allemagne a produit un investissement de plus de 4.5 millions de dollars ${ }^{6}$ du mois de janvier au mois de mai. L'expectative est de voir l'investissement en publicité arriver presque aux deux millions de dollars seulement pour le mois de juin ».

Quant aux aspects économiques, la publicité prétend agir sur le comportement des gens, en prenant appui sur leurs représentations, (leurs dénotations et leurs connotations).

6 La Banque du gouvernement avait fixé ce jour l'échange en 510,06 pour l'achat colon/dollar et 512,53 pour la vente. Consulté en $:<$ http://indicadoreseconomicos.bccr.fi.cr/indicadoreseconomicos/Cuadros/ frmVerCatCuadro.aspx?CodCuadro=400\&Idioma=1\&FecInicial=2006/06/14\&FecFinal=2006/06/14\&>. 


\section{« Lecture » de la publicité}

L'importance de lire ou d'interpréter la publicité est multiple, car cette compréhension nous permet d'accéder aux messages implicites ainsi qu' aux aspects culturels qui sont introduits par les publicitaires. $C^{\prime}$ est une façon d'approcher la culture et la civilisation d'un peuple, en découvrant l'intertextualité.

Particulièrement, quand on " lit » une image publicitaire puisqu'elle est chargée d'un message de contenu économique, il est important d'envisager l'analyse sur deux axes : celui du vocabulaire et de la linguistique ; et celui de la sémantique de l'image, qui nous permettra d'associer langage visuel-langue-culture, pour une interprétation plus approfondie.

Samayoa mentionne les fins diverses associées à la publicité, qui vont au-delà du commercial:

Je suis absolument d'accord avec le fait que la publicité n'a pas seulement des fins commerciales, mais aussi politiques, sociales, morales et idéologiques. La publicité est considérée comme la « langue dominante » de la culture, une langue sociale, sans doute le système d'information le plus important de l'histoire parce que la publicité peut toucher tous les secteurs et tous les domaines, et comme nous avons vu auparavant, elle est présente dans tous les moyens de communication. La publicité peut aussi être considérée comme l'écho de psychologies différentes, puisqu' elle nous montre les diverses gammes de produits consommés et les tendances des individus vers la promotion de ces produits. ${ }^{7}$

À travers la « lecture » de la publicité, nous pouvons aussi approcher certains éléments linguistiques qui nous permettront de revenir sur 1'étude de la langue tels que les adjectifs, les modes verbaux, la rhétorique...

7 Krishna Julieta Samayoa Ramírez, La publicité représente-t-elle la société et la culture d'un pays? (Puebla, MX : Universidad de las Américas, 2005) 80. 
L'expérience personnelle, après presque une dizaine d'années de travail, y comprise une période comme inspectrice du MEP ; joint aux échanges entre collègues lors des formations, a permis de confirmer que une analyse publicitaire se limitait à décomposer les publicités selon le schéma de Jakobson.

Pourtant, le langage polysémique des images et la charge socio-culturelle qu'il porte (à la fois figuration, représentation, mais aussi source créée), nous semblait motif suffisant pour développer cette recherche, puisque l'interprétation de cette polysémie non seulement enrichit les perceptions personnelles, mais dérive en même temps vers un tourbillon de possibilités d'expression, de compréhension et d'échange, indispensables à tout enseignement linguistique et culturel. Pour cette interprétation, l'analyse du discours publicitaire constitue l'outil privilégié vers une pensée d'éthique, d'esthétique et de citoyenneté.

\section{Contenus sémiologiques de l'image et suggestion publicitaire}

La publicité est une forme de communication dont le but est de fixer l'attention du consommateur sur un objet, et de l'inciter à adopter un comportement déterminé vis-à-vis de ce dernier (techno-sciences.net)

Parmi les classifications de la publicité, nous allons retenir le concept de publicité suggestive, proposée en 1911 par Gérin et Espinadel et reprise des années après par Bya (1973), où l'influence de la deuxième topique psychanalytique de Freud (1923) a permis de définir l'accent sur l'image, le pouvoir qu'elle entraîne.

La publicité est séductrice. Elle cherche à attirer l'attention des clients potentiels bien ciblés, pour les convaincre d'acheter un produit déterminé. Elle incite à la consommation d'un produit ou d'une marque particulière. Pour parvenir à son but, elle aura recours au côté caché des images, avec leurs significations, leurs contenus sémiologiques et représentatifs. Lambert (1994) l'explique en disant 
que « l'image est toujours une langue étrangère, l'image n'est pas un langage universel. C'est à partir de ce premier principe que peut se construire une sémiologie de l'image dès lors que cette dernière est considérée comme un fait de culture et non pas un reflet de nature » (Recherches et applications LFDM, $1994: 35$ ).

Les images apparaissant dans un dictionnaire enfantin, par exemple, et qui traduisent le signe proprement dit, n'ont pas le même signifiant dans des contextes différents : une pomme, par exemple, dans un livre de vocabulaire pour les petits ne veut pas dire la même chose que la pomme dans le couvercle d'un ordinateur.

Comme dit Roland Barthes (1964: 1), si naturellement toutes les images portent une charge de sens, " en publicité, la signification de l'image est assurément intentionnelle (...) ». C'est pour cela qu'il nous faut aussi parler des "pré-construits culturels » (PCC), puisqu'ils seront liés directement à la situation de communication qui va permettre aux interlocuteurs de passer par le tamis pour filtrer et sélectionner ceux qui conviennent à la situation.

Les variables socio-culturelles peuvent être déterminantes dans les processus de production-réception des énoncés. Parlons, par exemple de la publicité de LU, « Hommage à Van Gogh ». Issue pour commémorer le centenaire de la mort du peintre, la marque LU, fabricante des plusieurs biscuits, ont publié une image où l'un des plus fameux de ses biscuits, le petit beurre à forme traditionnellement rectangulaire, apparaissait avec un coin craqué.

Pour arriver à comprendre la relation entre Vincent Van Gogh et le Petit Beurre au coin craqué, c'est indispensable de posséder des PCC : il faut savoir, par exemple, que le peintre hollandais, dans une crise de folie s'est coupé l'oreille, puis, qu'il a peint l'autoportrait à l'oreille coupée (1889), et encore, il faut savoir que les quatre coins de ce biscuit sont connus par les enfants, en France, comme les « oreilles ».

Le support iconographique en classe s'inscrit bien sûr dans ce cadre de polysémie. En classe de langue peut-être encore plus. Si le professeur vise le développement de la pensée critique des élèves, il 
devra faire recours incontournable aux fonctions complexes de l'iconique, où se mélangent la découverte et les savoirs. L'interprétation de cette polysémie non seulement enrichit les perceptions personnelles, mais dérive en même temps vers un tourbillon de possibilités d'expression, de compréhension et d'échange, indispensables à tout enseignement linguistique et culturel. Pour cette interprétation, l'analyse du discours publicitaire constitue l'outil privilégié.

\section{L'analyse du texte publicitaire et le concept d'iconotexte}

L'analyse du discours en général, a pour objet les liens, les relations et les interdépendances entre l'organisation textuelle et la situation de communication. Il y a deux sortes d'analyse du texte que nous pouvons distinguer de cette façon : l'analyse du texte discursif et l'analyse du texte morphosyntaxique. Dans ce dernier, l'importance porte sur les constituants de la phrase. Dans l'analyse du texte discursif, par contre, ce qui est important c'est le sens, la place que les idées prennent dans l'esprit, pas dans la phrase. Elle se base sur les conditions d'interprétation et de production, prenant en compte la partie extralinguistique.

Or, nous n'allons pas faire l'analyse d'un discours quelconque, mais l'analyse du discours publicitaire, lequel est chargé de sens et d'éléments divers, autres, qui ne sont pas toujours trouvés dans le discours quotidien.

L'existence du discours publicitaire n'est plus mise en question, elle est reconnue comme un fait et maintenant elle se trouve à côté du discours journalistique, juridique, philosophique ou politique. Il y a sans doute, un secteur spécifique de production verbale suffisamment établi pour le constituer en une formation et en une pratique discursive.

L'analyse du discours publicitaire met en relation le tout et ses parties, car pour elle « la formation discursive est pensée d'un même mouvement comme contenu, comme mode d'organisation des hommes et comme réseau spécifique de circulation des énoncés » 
(Maingueneau $2002: 458$ ). Cette relation du tout et des parties nous invite à chercher un lien qui nous permet d'assembler les unités textuelles pour pouvoir enfin considérer l'analyse du discours publicitaire comme le produit résultant.

C'est là où nous allons emprunter le concept d'iconotexte, que le professeur Gilles Lugrin, définit comme un « objet discursif pluri-sémiotique ... la notion d'iconotexte désigne un message mixte, un ensemble formant une unité signifiante à part entière, dans laquelle le linguistique et l'iconique se donnent comme une totalité insécable, mais dans laquelle ils conservent chacun leur spécificité propre » (Lugrin 2006).

L'acception du terme d'iconotexte et son analyse, provoque une série de processus que nous feront aller et venir entre deux systèmes sémiologiques pour bien les interpréter.

La lecture de l'image et la lecture du textuel se mêleront parfois en un mécanisme de transfert des sens multiples appartenant aux deux systèmes. Parfois, ces mécanismes seront conscients, parfois non.

L'iconotexte publicitaire constitue alors « une unité compacte en plein dialogue, dont l'identité et l'autonomie du texte et de l'image sont en même temps préservées et mélangées, et forme une " totalité » signifiante, un énoncé de taille complète, subsumant de multiples unités (slogan, visuel, logo...). Mais le bien-fondé de cet « objet de sens » n'est cependant jamais définitivement acquis : le logo peut tout aussi légitimement être considéré comme un « texte » à part entière, comme un énoncé de taille complète » (Lugrin 2006).

Le jeu d'accommodation de l'œil et de l'esprit aux deux réalités différentes mais fondues dans un seul symbole nous emmènera finalement à découvrir le véritable sens sous-jacent de l'iconotexte publicitaire.

\section{Les signes et l'iconotexte:}

Pour commencer, nous pouvons expliquer que l'iconotexte publicitaire de presse écrite qui nous concerne, est composé d'un 206 
ensemble de signes de trois origines différentes : linguistiques, plastiques et iconiques. Ces signes sont physiquement regroupés et se complémentent pour arriver à un ensemble graphique qui sera finalement publié dans un média de presse écrite.

Parmi les signes d'origine linguistique, nous avons le slogan, le titre et le pavé rédactionnel. Quant aux signes iconiques, nous allons faire le point sur l'image, le « pack shot » et le visuel. Et finalement, par rapport aux signes plastiques, nous parlerons de la forme, la couleur, la texture, la typographie et les plans. Ces ressources seront mises au service des stratégies et utilisées pour atteindre les résultats voulus.

D'après Elena Ivranova-Glédel, dans son cours de linguistique appliquée à la publicité, nous pouvons classifier les signes linguistiques comme suit :

Le slogan de marque : c'est une formule brève, concise, et frappante. Il cherche à résumer l'esprit ou la philosophie de la marque en quelques mots. Il est souvent pourvu d'une structure rythmique qui reste bien dans la mémoire.

Il peut être en position de titre, appelé « d'accroche » et placé en début de l'annonce ; ou bien en position dite «d'assise », à la fin de l'annonce.

Le pavé rédactionnel : c'est l'explication ou l'argumentation que portent, de moins en moins, les publicités. Généralement mis en bas de page et en écriture plus petite que le reste de l'ensemble.

La marque : apparaît très souvent à côté du slogan.

Les signes iconiques:

L'image: c'est l'image choisie pour mettre en relief le produit. Elle peut être une photographie, un dessin, une peinture,... C'est la partie la plus importante car elle doit attirer l'attention. Selon Ivranova-Glédel, les publicités où domine 1 'image sont mémorisées par $41 \%$ de plus de lecteurs que les autres. 
Le pack shot : ce sont ces images où la publicité est écrite sur la prise de vue du gros plan du produit.

Le visuel : c'est le résultat du mélange de l'image et du texte. Dans son ensemble, il recherche l'effet de choc. L'image publicitaire travaillée à l'ordinateur est corrigée, arrangée, épurée et finalement transformée en visuel pour faire appel aux sens et éveiller les désirs.

Les signes plastiques:

Tant les signes linguistiques que les signes iconiques sont habillés, même manipulés par les signes plastiques.

La forme : c'est l'organisation de l'espace visuel, en accord avec la perception voulue. Elle suit, généralement, une structure qui relie la taille et la position à l'importance du signe montré. Elle prend en compte les objets, les couleurs, les textures et les plans.

La couleur: L'importance de la couleur est indiscutable, car il est démontré qu' $[\ldots]$ « en parcourant les publicités de la presse écrite (journaux, revues, magazines), le regard s'arrête sur les annonces en couleur pendant deux secondes, au grand maximum, alors que les annonces en noir et blanc ne retiennent l'attention que pendant un vingtième de seconde »; <http://leanantes.com/LICENCE3/Linguistique_Image.pdf $>$.

$\mathrm{C}^{\prime}$ est le premier aspect à observer. Elle établit un lien très fort avec les représentations culturelles. Si bien que des stéréotypes, certaines couleurs ne sont pas bien acceptées pour des produits masculins et à l'envers. Par contre, la créativité et l'originalité de l'utilisation de certaines couleurs peut aussi déclencher les ventes d'un produit.

La texture : Le Petit Robert (1986 : 1955) la décrit comme « Disposition des fils d'une chose tissée. Arrangement, disposition des éléments d'une matière. Arrangement de la matière et des pores a l'intérieur d'un solide divisé (en grains) ou poreux ». C'est elle qui donne l'aspect lisse, moiré, granuleux, ... aux images.

La typographie : c'est la stylisation de l'écriture. C'est la manière de se servir des caractères et le choix de la police, du corps et de la mise en page. Elle sert à exprimer à l'écrit ce qui serait les inflexions 
de la voix à l'oral, voilà la raison de son rapport très étroit avec l'intonation, le volume, et l'expression que l'on veut donner au texte.

Les plans : grâce à la position des objets dans les différents plans, l'importance relative des éléments pourra être interprétée et mise en relief. Les différents plans d'une image permettent de saisir ce qui a été privilégié parce que c'est ce qui est porté au devant de la scène. Tous ces éléments participent à la hiérarchisation des unités linguistiques. La lisibilité de l'image sera conséquente avec la position et l'importance des unités entre elles et dans l'ensemble.

Les unités linguistiques, iconiques et plastiques, convergent et se complètent. Elles peuvent même s'opposer les unes aux autres, comme les fils dans l'envers d'une broderie. Quand on le retourne, chaque élément prend sa place et retrouve le sens particulier et le sens global. Voilà pourquoi la description des relations entre les différentes unités constitue le point de départ de l'analyse de l'iconotexte publicitaire.

\section{Composition de la publicité}

La publicité est composée, fondamentalement, de quatre éléments d'action : la communication, le produit, le prix et la distribution. Ces éléments seront mis au service de la stratégie de marketing des entreprises afin d'atteindre leurs buts commerciaux, que nous pourrions schématiser comme suit :

a) Faire connaître un nouveau produit dans le but de le vendre.

b) Accroître la consommation d'un produit déjà existant.

c) Capturer la fidélité de la clientèle.

d) Stabiliser les ventes en diminuant l'effet saisonnier.

Le message de la publicité s'intéresse à faire voir dans le produit ou la marque proposés, la promesse de la satisfaction du « besoin » créé. Pour cela, il développe des stratégies diverses selon le 
produit, le milieu, le besoin et le but poursuivi. Nous pouvons mentionner parmi les plus courantes : le témoignage des personnages célèbres ; les propositions d'offres exceptionnelles et immédiates ; l'évocation des désirs, des réalisations de projets ou des voyages ; la manipulation des statistiques ; l'exploitation d'expectations chez les enfants ; et aussi, malheureusement, nous ne pouvons laisser de côté la mention de l'exploitation de la femme en tant qu'objet sexuel.

Dès les années soixante, le psychologue Robert Zajonc (1968), avait lui aussi ajouté une autre stratégie très importante dans l'exécution de la publicité, exprimée dans sa théorie de «l'effet d'exposition répétée » (mere-exposure effect) laquelle soutient que l'on peut « former et modifier les préférences et réponses affectives des sujets vers des stimulus comme conséquence de sa présentation répétée " $<\mathrm{http}: / /$ www.psicothema.com/psicothema.asp?id=376> Aceptado el 18 de febrero de 2000/ 23 sept. 2012). Autrement dit, le fait de répéter un message le rend familier et améliore son image vers une acceptation plus positive.

\section{Caractéristiques de l'étude et du dispositif}

La recherche que nous présentons possède un format mixte, à dominance qualitative. L'étude réalisée prétend harmoniser les données obtenues du diagnostic avec les propositions des théoriciens sélectionnés, pour aboutir à une proposition concrète : un dispositif pour l'entraînement à l'analyse du discours, envisagé dès un niveau encore classé comme débutant.

L'étude diagnostique est basée sur une triangulation de données à dominance aussi qualitative, mais avec l'appui d'un sondage dont les données recueillies ont été traitées de manière quantitative. Cette étude a regroupé l'analyse des contenus du programme d'études, une enquête aux enseignants et une confrontation de diverses théories sur la communication, le langage et la publicité, pour la synthèse des concepts de base. L'enquête réalisée à l'intention de 
sondage auprès des enseignants n'a pas voulu être représentative de la population, car l'échantillon n'a pas été choisi par des moyens statistiques, mais par convenance.

Le dispositif a été formulé à partir de la synthèse des études diagnostiques menées et a aboutit à la proposition d'une séquence de fiches pour l'analyse iconotextuelle.

\section{Contextes social, géographique et temporel de la mise en pratique du dispositif}

La recherche a été mise en place dans deux collèges de la région d'Alajuela, les deux dans le canton de Poas : Lycée Carrillos de Poas et au Lycée de Poas, au cours de trois années scolaires consécutives : 2010, 2011 et 2012, auprès d'adolescents de la 10 année, ayant acquis le niveau A1 du CECRL.

\section{Proposition du dispositif}

Le dispositif proprement dit consiste en une séquence d'activités pour l'analyse iconotextuelle, conformant un ensemble de séances. Cet ensemble de séances possède des objectifs, des activités de médiation et des activités d'évaluation qui lui sont propres en dépendant de chaque séance.

L'ensemble est conçu en séances séquentielles organisées autour de trois étapes :

- Sensibilisation

- Analyse des iconotextes

- Production

La sensibilisation est une étape où les apprenants prendront conscience de l'influence de la publicité dans leurs vies.

Dans l'analyse des iconotextes, nous pouvons distinguer trois moments aussi séquentiels. 
Un premier moment proposera une analyse décortiquée de l'image. Nous avons déjà dit que l'image est la partie la plus attirante $\mathrm{d}^{\prime}$ un iconotexte et c'est pour cela que nous avons choisi de faire son analyse en premier. L'image publicitaire est le produit d'un travail de recherche qui a pour but d'évoquer chez le récepteur une émotion profonde, avec laquelle il doit s'identifier et identifier sa culture ellemême, pour éveiller ce sens d'appartenance. L'équipe de production peut se réunir pendant six mois, jusqu'à obtenir le produit final, voilà donc, l'importance de faire tout d'abord, l'analyse de l'image.

L'iconotexte est composé d'un ensemble de signes de trois origines différentes : linguistiques, plastiques et iconiques. L'analyse de l'image prendra en compte principalement les signes plastiques, c'est-à-dire, les couleurs, les formes, les plans, la typographie...

Un deuxième moment sera constitué par l'analyse des signes linguistiques apparaissant sur les textes publicitaires : le slogan, la marque, le pavé rédactionnel...

Un troisième moment abordera l'analyse communicative traditionnelle, inspirée sur le modèle de Jakobson.

Finalement viendra la production, conçue comme le moment où les apprenants mettront en pratique tous les acquis, sous la consigne de produire un iconotexte. Le travail se déroulera sous les conditions suivantes :

- Il doit y avoir au moins une image

- Il doit y avoir une marque

- Il doit y avoir un slogan

- Il doit y avoir un petit pavé rédactionnel

\section{Production iconotextuelle des apprenants}

Cette étape finale aboutit à un produit concret, permettant d'évaluer l'application. Les produits de cette étape ont été analysés pour vérifier l'assimilation du processus et l'application des savoirs à la construction de l'iconotexte. 


\section{Conclusions}

- $\quad \mathrm{D}^{\prime}$ après les produits iconotextuels obtenus dans la dernière étape d'application, le dispositif favorise et contribue à l'entraînement des jeunes dans l'analyse du discours.

- L'emploi de la technique et les éléments de construction d'iconotexte chez les élèves met en évidence l'acquisition des habilités d'analyse.

- Les jeunes ont besoin de se fournir de la connaissance élémentaire de l'analyse du discours comme un outil pour mieux prendre des décisions dans leurs vies, objectif pris en compte par le Gouvernement du Costa Rica, dans les programmes d'études au niveau national dans le cadre de l'éthique, l'esthétique et la citoyenneté.

- L'analyse de la publicité est un sujet de importance pour le développement des habilités de réflexion chez les élèves et son approche en classe de FLE devrait se modifier pour qu' il soit mieux compris et exploité par les collègues au profit des apprenants. 